\title{
LIST OF PARTICIPANTS
}

Akinian, S. T., Izmiran, Moscow, U.S.S.R.

Ambrož, P., Astronomical Institute, 25165 Ondřejov, C.S.S.R.

Antalová, A., Chair of Technical Physics, Technical University for Transport, 01088 Žilina, C.S.S.R.

Antonucci, E., Physical Institute, I-10125, Torino, Italy.

Anzer, U., Max-Planck-Institut, 8000 München, Föhringer Ring 6, F.R.G.

Artus, H., VEB Carl Zeiss, Jena, D.D.R.

Aurass, H., Observatorium für solare Radioastronomie, DDR-1501, Tremsdorf, D.D.R.

Balklavs, A. E., Radioastronomical Institute, Riga, Latvian S.S.R., U.S.S.R.

Beck, H. G., VEB Carl Zeiss, Jena, D.D.R.

Bednářová, B., Geophysical Institute, 14131 Praha, C.S.S.R.

Belvedere, G., Astrophysical Observatory, I-95125 Catania, Italy.

Blanco, C., Astrophysical Observatory, I-95125 Catania, Italy.

Böhme, A., Sonnenobservatorium Einsteinturm, 15 Potsdam, Telegrafenberg, D.D.R.

Bonov, A. D., University of Sofia, Sofia, Bulgaria.

Bruzek, A., Fraunhofer Institut, D 78 Freiburg im Breisgau, Schöneckstrasse 6, F.R.G.

Bumba, V., Astronomical Institute, 25165 Ondřejov, C.S.S.R.

Chistjakov, V. F., Service of Sun, Ussurijsk 692533, U.S.S.R.

Chvojková, E., Astronomical Institute, 12023 Praha, C.S.S.R.

Cimakhovich, N. P., Radioastronomical Institute, Riga, Latvian S.S.R., U.S.S.R.

Csada, I. K., Konkoly Observatory, Konkoly-Thege U. 13-17, 1525 Budapest, XII., Hungary.

Dara-Papamargariti, H., Research Centre for Astronomy and Applied Mathematics, 14 Anagnostopoulou Street, Athens (136), Greece.

Deinzer, W., Universitäts-Sternwarte, D-34 Göttingen, Geismarlandstrasse 11, F.R.G.

Demkina, L. B., Izmiran, Moscow, U.S.S.R.

Deubner, F. L., Fraunhofer Institut, Schöneckstrasse 6, 7800 Freiburg im Breisgau, F.R.G.

Dezsö, L., Heliophysical Observatory, H-4010 Debrecen, P.O. Box 30, Hungary.

Dravins, D., Lund Observatory, Svanegatan 9, S-222 24, Lund, Sweden.

Durney, B. R., National Center for Atmospheric Research, P.O. Box 1470, Boulder, Colo. 80302, U.S.A.

Durrant, Ch.-J., Fraunhofer Institut, Schöneckstrasse 6, D-78 Freiburg im Breisgau, F.R.G.

Elwert, G., Lehrstuhl für Teoretische Astrophysik, 7400 Tübingen, Auf der Morgenstelle 10, F.R.G. 
Fárník, F., Astronomical Institute, 25165 Ondřejov, C.S.S.R.

Fischer, S., Astronomical Institute, 12023 Praha, C.S.S.R.

Fortini, T., Astronomical Observatory, 00136 Roma, Italy.

Fossat, E., Astrophysical Institute, 98bis Boulevard Arago, Paris 14e, France.

Gelfreikh, G. B., Main Astronomical Observatory, Pulkovo, 196140 Leningrad, U.S.S.R.

Ghabrus, R. A., Helwan Observatory, Helwan, near Cairo, U.A.R.

Gigolashvili, M. S., Abastumani Astrophysical Observatory, Abastumani, Georgia, U.S.S.R.

Gilman, P. A., Advanced Study Program, NCAR, P.O. Box 3000, Boulder, Colo. 80302, U.S.A.

Giovanelli, R. G., CSIRO, National Standards Laboratory, Division of Physics, University Grounds, City Road, Chippendale, N.S.W., 2008, Sydney, Australia.

Gleissberg, W. K. H., Senckenberganlage 23, 6000 Frankfurt, F.R.G.

Gnevyshev, M. N., Astronomical Observatory, Pulkovo, 196140 Leningrad, U.S.S.R.

Gnevysheva, R. S., Astronomical Observatory, Pulkovo, 196140 Leningrad, U.S.S.R.

Godoli, G., Astrophysical Observatory, I-95125 Catania, Italy.

Golub, L., American Science and Engineering, Inc., 955 Massachusetts Avenue, Cambridge, Mass. 02139, U.S.A.

Grigoriev, V. M., Sibizmiran, Irkutsk 664033, U.S.S.R.

Gurtovenko, Main Astronomical Observatory, Kiev, U.S.S.R.

Gutcke, H., VEB Carl Zeiss, Jena, D.D.R.

Halenka, J., Geophysical Institute, 14131 Praha, C.S.S.R.

Hamatschek, R., VEB Carl Zeiss, Jena, D.D.R.

Hartmann, R., Astronomical Institute, Senckenberg-Angle 23, 600 Frankfurt/Main 1, F.R.G.

Hedeman, E. R., McMath-Hulbert Observatory, 895 N. Lake Angelus Road, Pontiac, Michigan 48055, U.S.A.

Hejna, L., Astronomical Institute, 25165 Ondřejov, C.S.S.R.

Howard, R., Hale Observatories, 813 Santa Barbara Street, Pasadena, Calif. 91101 , U.S.A.

Jäger, F. W., Zentralinstitut für Solar-Terrestrische Physik, DDR-15 Potsdam, Telegrafenberg, D.D.R.

Jakimiec, J., Astronomical Institute, Kopernika 11, Wroclaw, 51-622, Poland.

Karabin, M., Astrophysical Department, Faculty of Sciences, Studentski trg 16, P.O.

Box 550, 11001 Beograd, Yugoslavia.

Kasinskij, V. V., Sibizmiran, Irkutsk 664033, U.S.S.R.

Kato, S., University of Kyoto, Kyoto, Japan.

Khetsuriani, Abastumani Astrophysical Observatory, Abastumani, Georgia, U.S.S.R.

Kim, I. S., Izmiran, Moscow, U.S.S.R.

Kleczek, J., Astronomical Institute, 25165 Ondřejov, C.S.S.R.

Klvaňa, M., Astronomical Institute, 25165 Ondřejov, C.S.S.R.

Knoška, Š., Astronomical Institute, 05960 Tatranská, Lomnica, C.S.S.R. 
Kopecký, M., Astronomical Institute, 25165 Ondřejov, C.S.S.R.

Kotrč, P., Astronomical Institute, 25165 Ondřejov, C.S.S.R.

Kovács, A., Heliophysical Observatory, P.O. Box 30, H-4010, Debrecen, Hungary.

Krause, F., Zentralinstitut fur Astrophysik, 15 Potsdam, Telegrafenberg, D.D.R.

Křivský, L., Astronomical Institute, 25165 Ondřejov, C.S.S.R.

Krüger, A., Zentralinstitut für Solar-Terrestrische Physik, 1199 Berlin-Adlershof, Rudower Chaussee 5, D.D.R.

Kubičela, A., Astronomical Observatory, Volgina 7, 11050 Beograd, Yugoslavia.

Kuklin, G. V., Sibizmiran, Irkutsk 664033, U.S.S.R.

Kulidzhanishvili, V. I., Abastumani Astrophysical Observatory, Abastumani, Georgia, U.S.S.R.

Künzel, H., Sonnenobservatorium Einsteinturm, 15 Potsdam, Telegrafenberg, D.D.R.

Lê-Bach-Yén, Astronomical Institute, 25165 Ondřejov, C.S.S.R.

Leftus, V., Astronomical Institute, 25165 Ondřejov, C.S.S.R.

Lielausis, O. A., Astronomical Observatory, Riga, Latvian S.S.R., U.S.S.R.

Macák, P., Astronomical Institute, 25165 Ondřejov, C.S.S.R.

Macháček, M., Astronomical Institute, 25165 Ondřejov, C.S.S.R.

Macris, C. J., Research Center for Astronomy and Applied Mathematics, 14 Anagnostopoulou Street, Athens (136), Greece.

Marilli, E., Astrophysical Observatory, I-95125 Catania, Italy.

Mestel, L., Astronomy Centre, Physics Building, University of Sussex, Falmer, Brighton BN1 9QH, England.

Mogilevskij, E. I., Izmiran, Moscow, U.S.S.R.

Motta, S., Astrophysical Observatory, I-95125 Catania, Italy.

Newkirk, G. A., Jr., N.C.A.R., High Altitude Observatory, P.O. Box 1470, Boulder, Colo. 80302, U.S.A.

Nită, I., Observatoire de Bucarest, 5 rue Cutitul de Argint, Bucarest 28, Roumania.

Noci, G., Astrophysical Observatory Arcetri, Largo E. Fermi 5, 50125 Firenze, Italy.

Obridko, V. N., Izmiran, Moscow, U.S.S.R.

Oetken, L., Zentralinstitut für Astrophysik, 15 Potsdam, Telegrafenberg, D.D.R.

Paciorek, J. M., Astronomical Observatory, Kopernika 11, 51-622 Wroclaw, Poland.

Pallavicini, R., Astrophysical Observatory Arcetri, Largo E. Fermi 5, 50125 Firenze, Italy.

Paluš, P., Faculty of Sciences, University Bratislava, Trnavská Str. 1, 80000 Bratislava, C.S.S.R.

Parker, E. N., Laboratory for Astrophysics and Space Research, 933 East 56th Street, Chicago, Ill. 60637, U.S.A.

Paterno, L., Astrophysical Observatory, I-95125 Catania, Italy.

Pflug, K., Zentralinstitut für Solar-Terrestrische Physik, Sonnenobservatorium Einsteinturm, 15 Potsdam, Telegrafenberg, D.D.R.

Pirronello, V., Astrophysical Observatory, I-95125 Catania, Italy.

Pospíšil, M., Astronomical Institute, 25165 Ondřejov, C.S.S.R. 
Priest, E. R., Department of Applied Mathematics, North Haugh, St. Andrews, KY16 9SS, U.K.

Rädler, K.-H., Zentralinstitut für Astrophysik, 15 Potsdam, Telegrafenberg, D.D.R.

Rajský, V., Astronomical Institute, 12023 Praha, C.S.S.R.

Rodonò, M., Astrophysical Observatory, I-95125 Catania, Italy.

Rompolt, B., Astronomical Observatory, Kopernika 11, 51-622 Wroclaw, Poland.

Roxburgh, I. W., Queen Mary College, University of London, Department of Applied Mathematics, Mile End Road, London E1 4NS, England.

Rüdiger, G., Zentralinstitut für Astrophysik, 15 Potsdam, Telegrafenberg, D.D.R.

Rušín, V., Astronomical Institute, 05960 Tatranská Lomnica, C.S.S.R.

Ruždjak, V., Institute of Physics of the University, P.O. Box 304, 41001 Zagreb, Yugoslavia.

Ružičková-Topolová, B., Astronomical Institute, 25165 Ondřejov, C.S.S.R.

Rybanský, M., Astronomical Institute, 05960 Tatranská Lomnica, C.S.S.R.

Schröter, E. H., Universitäts-Sternwarte, D-34 Göttingen, Geismarlandstrasse 11, F.R.G.

Shmeleva, O. P., Izmiran, Moscow, U.S.S.R.

Šidlichovský, M., Astronomical Institute, 12023 Praha, C.S.S.R.

Slottje, C., Radio Observatory, Dwingeloo, The Netherlands.

Smith, D. F., N.C.A.R., High Altitude Observatory, P.O. Box 3000, Boulder, Colo. 80302, U.S.A.

Staude, J., Zentralinstitut für Solar-Terrestrische Physik, Sonnenobservatorium Einsteinturm, 15 Potsdam, Telegrafenberg, D.D.R.

Stenflo, J. O., Lund Observatory, Svanegatan 9, S-222 24, Lund, Sweden.

Stepanian, N. N., Crimean Astrophysical Observatory, Nauchny, Crimea 334413, U.S.S.R.

Stepanov, V. E., Sibizmiran, Irkutsk 664033, U.S.S.R.

Stix, M., Universitäts-Sternwarte, 3400 Göttingen, Geismarlandstrasse 11, F.R.G.

Suda, J., Astronomical Institute, 25165 Ondřejov, C.S.S.R.

Sýkora, J., Astronomical Institute, 05960 Tatranská Lomnica, C.S.S.R.

Tlamicha, A., Astronomical Institute, 25165 Ondřejov, C.S.S.R.

Tritakis, B., Research Center for Astronomy and Applied Mathematics, 14 Anagnostopoulou Street, Athens (136), Greece.

Touminen, J. V., Observatory and Astrophysics Laboratory, Tähtitorninmäki, SF-00130, Helsinki 13, Finland.

Vainshtein, S. I., Sibizmiran, Irkutsk 664033, U.S.S.R.

Valníček, B., Astronomical Institute, 25165 Ondřejov, C.S.S.R.

Vandakurov, Yu. V., Physical and Technical Institute A. F. Ioffe, Leningrad, 194021, U.S.S.R.

Vertlib, A. B., Sibizmiran, Irkutsk 664033, U.S.S.R.

Vitinskij, Yu. I., Main Astronomical Observatory, Pulkovo, Leningrad, 196140, U.S.S.R.

Vujnović, V., Institut of Physics of the University, P.O. Box 304, 41001 Zagreb, Yugoslavia. 
Wagner, W. J., Sacramento Peak Observatory, Air Force Cambridge Research Laboratories, Sunspot, N.M. 88349, U.S.A.

Weiss, N. O., Department of Applied Mathematics and Theoretical Physics, Silver Street, Cambridge, CB3 9EW, England.

Wilcox, J. M., Institute for Plasma Research, Via Crespi, Stanford, Calif. 94305, U.S.A.

Yoshimura, H., Hale Observatories, California Institute of Technology, 813 Santa Barbara Street, Pasadena, Calif. 91101, U.S.A.

Zappala, R., Astrophysical Observatory, I-95125 Catania, Italy.

Zwaan, C., Astronomical Observatory 'Sonnenborgh', Zonnenburg 2, Utrecht, The Netherlands. 